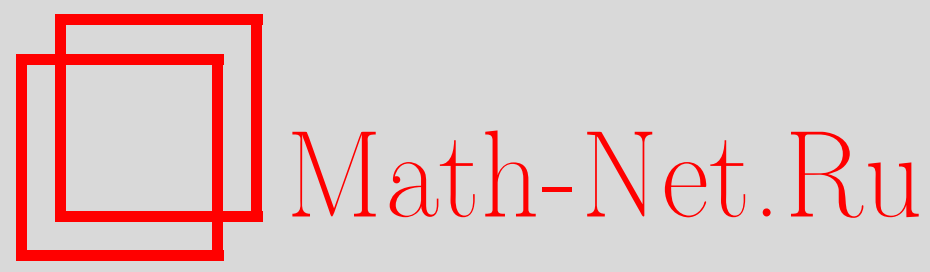

В. В. Кабанов, А. А. Махнев, Д. В. Падучих, О графах без корон с регулярными $\mu$-подграфами, II, Матем. заметки, 2003, том 74, выпуск 3, 396-406

DOI: https://doi.org/10.4213/mzm273

Использование Общероссийского математического портала Math-Net.Ru подразумевает, что вы прочитали и согласны с пользовательским соглашением http://www.mathnet.ru/rus/agreement

Параметры загрузки:

IP: 54.84 .234 .179

26 апреля 2023 г., $12: 10: 26$

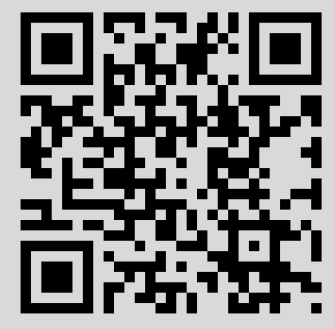




\title{
О ГРАФАХ БЕЗ КОРОН С РЕГУЛЯРНЫМИ $\mu$-ПОДГРАФАМИ, ІІ
}

\author{
В.В. Кабанов, А. А. Махнев, Д. В. Падучих
}

Короной называется полный многодольный граф̆ $K_{1,1,3}$. В работе изучаются графы Тервиллигера без корон и графы без 3-коклик с регулярными $\mu$-подграфами заданной положительной степени. Как следствие, выяснено локальное строение графов, в которых окрестности вершин являются регулярными граффами Тервиллигера диаметра 2 , и некоторая из этих окрестностей не содержит 7 -лап. Получено описание связных графов без корон, в которых $\mu$-подграфы являются реберно регулярньми графами диаметра, не большего 2, с заданными параметрами.

Библиография: 6 названий.

Введение. Мы рассматриваем неориентированные графы без петель и кратных ребер. Для вершины $a$ графа $\Gamma$ через $\Gamma_{i}(a)$ обозначим подграф, индуцированньй $\Gamma$ на множестве всех вершин, находящихся на расстоянии $i$ от $a$. Подграф $[a]=\Gamma_{1}(a)$ назьвается окрестностью вершины $a$. Пусть $a^{\perp}-$ шар радиуса 1 с центром $a$. Для подграфа $A$ графа $\Gamma$ через $A^{\perp}$ обозначим $\bigcap_{a \in A} a^{\perp}$.

Через $k_{a}$ обозначим степень вершины $a$, т.е. число вершин в [a]. Граф $\Gamma$ назьвается регулярным валентности $k$, если $k_{a}=k$ для любой вершины $a$ из Г. Для ребра $\{a, c\}$ графа $\Gamma$ через $\lambda_{a c}$ обозначим число вершин в графе $[a] \cap[c]$. Граф $\Gamma$ назьвается реберно регулярным с параметрами $(v, k, \lambda)$, если $\Gamma$ - регулярньй граф степени $k$ на $v$ вершинах, в котором каждое ребро лежит точно в $\lambda$ треугольниках $\left(\lambda_{a c}=\lambda\right.$ для любого ребра $\{a, c\})$. Подграф, индуцированньй на $[a] \cap[b]$, назовем $\mu$-подграфом, если вершины $a, b$ находятся на расстоянии 2 , а через $\mu_{a b}$ обозначим число вершин в этом подграфе. Скажем, что $\mu(\Gamma)=\mu$, если для любых вершин $a, b$ с условием $d(a, b)=2$ верно равенство $\mu_{a b}=\mu$. Кореберно регулярным графом $с$ параметрами $(v, k, \mu)$ назьвается регулярньй граф степени $k$ диаметра 2 на $v$ вершинах с $\mu(\Gamma)=\mu$. Реберно регулярньй граф с параметрами $(v, k, \lambda)$ и $\mu(\Gamma)=\mu$ называется вполне регулярным графом с параметрами $(v, k, \lambda, \mu)$. Вполне регулярньй граф диаметра 2 назьвается сильно регулярным. Далее, подграф из Г будет означать индуцированный подграф.

Пусть $K_{m_{1}, \ldots, m_{n}}-$ полньй многодольньй граф с долями порядков $m_{1}, \ldots, m_{n}$. Если $m=m_{1}=\cdots=m_{n}$, то граф обозначается $K_{n \times m}$. Граф $K_{1, m}$ с $m \geqslant 3$ назьвается $m$-лапой (лапой, если $m=3$ ). Граф $K_{1, \ldots, 1, m}$ с $n$ долями порядка 1 назьвается $(n, m)$-короной ( $n$-короной, если $m=3 ;$ короной, если $n=2)$. Граф Г назовем слабо

Работа выполнена при финансовой поддержке Российского фонда фундаментальных исследований, грант № 02-01-00772. 
редуцированным, если из равенства $a^{\perp}=b^{\perp}$ следует $a=b$. Граф Г назовем редуцированным, если из включения $a^{\perp} \subseteq b^{\perp}$ следует $a=b$. Через $\Gamma^{\perp}$ обозначим подграф на множестве всех вершин $a$ таких, что $a^{\perp}=\Gamma$.

Графф на множестве пар $X \times Y$ называется $(m \times n)$-графом, если $|X|=m,|Y|=n$, а пары $\left(x_{1}, y_{1}\right)$ и $\left(x_{2}, y_{2}\right)$ смежны тогда и только тогда, когда $x_{1}=x_{2}$ или $y_{1}=y_{2}$. Иногда $(m \times n)$-граф назьвается прямоугольной решеткой. При $m=n$ граф назьвается решетчатым. Треугольным графом $T(m)$ назьвается граф с множеством неупорядоченных пар из $X$ в качестве вершин; $|X|=m$ и пары $\{a, b\},\{c, d\}$ смежны только, если они имеют единственный обший элемент.

Граф диаметра $d$ назьвается антиподальным, если отношение-совпадать или находиться на расстоянии $d$ - является отношением эквивалентности на множестве вершин графа. Антиподальный граф диаметра 3 на 12 вершинах, у которого окрестность любой вершины пятиугольник, назовем графом икосаэдра.

Под кликовым расиирением графа Г будем понимать граф, полученньй заменой каждой вершины $a$ из $Г$ на клику $(a)$, причем вершины из $(a)$ и $(b)$ смежны тогда и только тогда, когда $a$ и $b$ смежны в $\Gamma$. Кликовое расширение графа будем назьвать $\beta$-расширением, если каждая клика $(a)$ состоит из $\beta$ вершин.

Граф Тервиллигера - это неполный граф, в котором для любых двух вершин $a, b$, находяшихся на расстоянии 2 , подграф $[a] \cap[b]$ является $\mu$-кликой.

В работе [1] М. Нумата получил классификацию графов без корон, в которых $\mu$-подграфы являются изоморфными реберно регулярными графами диаметра 2 (естественно, не имеющими 3-лап). В связи с этим результатом представляет интерес следующая

ПроБЛЕМА А. Изучить графы, в которых каждый $\mu$-подграф является реберно регулярным графом без 3-лап с заданными параметрами $\left(v^{\prime}, k^{\prime}, \lambda^{\prime}\right)$.

В работе В. В. Кабанова [2] получено обобщение результата Нуматы, опирающееся на описание редуцированных графов без 3-лап с регулярными $\mu$-подграфами заданной положительной степени $\alpha$ (А. А. Махнев [3]) и классификацию графов без 3-лап, в которых $\mu$-подграфы имеют фиксированное число вершин (Махнев, Кабанов [4]).

Связный редуцированный граф без корон, содержаший 3-коклику, в котором н-подграфы регулярны заданной полохительной степени а и имеют диаметр 2, является графом Грассмана, графом Джсонсона или его частным, локально треугольным графом или графом Госсета.

Важным этапом в доказательстве этого результата явилась лемма 2.1 [2], в которой доказано, что слабо редуцированньй граф без 3-лап, содержащий 3-коклику, в котором $\mu$-подграфы регулярны заданной положительной степени, является редуцированньм.

ПРЕДЛОЖЕНИЕ. Если Г - связный слабо редуиированный әраф без 3-коклик, в котором н-подграфы регулярны степени $\alpha>0$, то Г является редуцированным графом или пятиугольной пирамидой.

В теореме 1 [4] доказано, что если Г - связньй граф Тервиллигера без 3-лап, то либо $\Gamma$ является кликовым расширением графа икосаэдра, либо подграф, индуцированньй на множестве всех вершин из $\Gamma-\Gamma^{\perp}$ с некликовыми окрестностями, является кликовым расширением пустого графа, клики или графа с $\mu=1$. 
Теорема 1. Пусть Г - связный граф Тервиллигера без корон, $\Gamma^{\prime}-$ подграф на множестве вершин с некликовыми окрестностями. Тогда выполняется одно из следующих утвержсдений:

(1) Г не содержит 3-коклик и является кликовым расширением 2-пути, 3-пути, пятиугольника или пятиугольной пирамиды;

(2) Г не содержит 3-лап, но содержит 3-коклику, диаметр Г больше 2 и либо

(a) Г является кликовым расширением графа икосаэдра, либо

(b) $\Gamma^{\prime}$ является кликой $и$ я является кликовым расширением графа $\Delta$, где $\Delta$ содержит $\delta$-клику и $\delta$ или $\delta-1$ вершин степени 1 , смехних $с$ различными вериинами клики, либо

(c) $\Gamma^{\prime}$ является кликовым расширением графа с $\mu=1$;

(3) $Г$ содержит 3-лапу, $\mu=1$ и окрестность любой вериины из $Г$ состоит из изолированных клик.

СЛЕДСТВИЕ 1. Пусть Г - связный граф, в котором окрестности вериин являются регулярными графами Тервиллигера диаметра 2. Если окрестность некоторой вериины не содержит 7-лап, то

(1) либо Г является кликовым расширением графа икосаэдра, либо окрестность каждой вершины из Г является кликовым $\beta$-расширением графа Петерсена;

(2) если окрестность некоторой вершинь из Г не содержит корон, то Гкликовое расиирение графа икосаэдра, граф $\overline{T(7)}$, граф Конвея-Смита на 63 вершинах диаметра 4 (это антиподальное 3 -накрытие $\overline{T(7)}$ ) или граф Доро на 65 вершинах диаметра 3.

Следующий результат является аналогом теоремы 3 из [2] для редуцированных графов без 3-коклик.

ТЕОрема 2. Пусть Г - связный редуиированный граф без 3-коклик, в котором каждый $\mu$-подграф регулярен заданной положительной степени $\alpha$. Тогда Г сильно регулярен.

Обратно, если $\Gamma$ - сильно регулярньй граф без 3-коклик с параметрами $(v, k, \lambda, \mu)$, то каждый $\mu$-подграф регулярен степени $\alpha=2 \lambda+2-k$.

СлЕДСТВИЕ 2. Пусть Г - связный редуиированный регулярный граф без корон, в котором каждый $\mu$-подграф реберно регулярен с заданными параметрами $\left(v^{\prime}, k^{\prime}, \lambda^{\prime}\right), \quad k^{\prime}>0$ и имеет диаметр не больше 2. Тогда выполняется одно из следуюших утверждений:

(1) Г не содержит 3-коклик и Г является либо октаэдром или треугольным графом $T(5)$, либо сильно регулярным графом с параметрами $\left(\left(s^{2}+3 s\right)^{2}\right.$, $\left.\left(s^{2}+2 s-1\right)\left(s^{2}+3 s+1\right),(s+2)\left(s^{3}+2 s^{2}-1\right),\left(s^{2}+2 s\right)\left(s^{2}+2 s-1\right)\right)$, либо нетривиальным кликовым расширением пятиугольника;

(2) Г содержит 3-коклику, не содержит 3-лап и является кликовым расиирением графа икосаэдра, треугольным графом $T(m), m \geqslant 6$, или графом Шлефли;

(3) Г содержит 3-лапу и является графом Грассмана, графом Джсонсона или его частным, локально треугольным графом или графом Госсета. 
Граф Г из пункта (2) заключения следствия 2 является дополнительньм для сильно регулярного графа $\Sigma$ без треугольников с параметрами $\left(\left(s^{2}+3 s\right)^{2}, s^{3}+3 s^{2}+s, 0, s^{2}+s\right)$. В этом случае достигается равенство в условии Крейна для $\Sigma$ и антиокрестность вершины в $\Sigma$ также является сильно регулярным графом с параметрами $\left(\left(s^{2}+2 s-1\right)\left(s^{2}+\right.\right.$ $\left.3 s+1), s^{2}(s+2), 0, s^{2}\right)$. Более того, по теореме 2 каждый $\mu$-подграф из $Г$ является сильно регулярным, поэтому для смежных вершин $a, b \in \Sigma$ подграф $\Sigma-\left(a^{\perp} \cup b^{\perp}\right)$ является сильно регулярным с параметрами $\left(\left(s^{2}+2 s\right)\left(s^{2}+2 s-1\right), s^{3}+s^{2}-s, 0, s^{2}-s\right)$. Хорошо известны существование и единственность $\Sigma$ в случаях $s=1$ (это дополнительньй граф для графа Клебша с параметрами $(16,5,0,2))$ и $s=2$ (граф Хигмена-Симса с параметрами $(100,22,0,6))$. Граф $\Sigma$ не существует в случае $s=3$ (см. главу 8 [6]). Ничего не известно о сушествовании таких графов для $s \geqslant 4$. Представляет интерес

ПроБЛЕмА В. Существует ли сильно регулярный граф с параметрами $\left(\left(s^{2}+\right.\right.$ $\left.3 s)^{2}, s^{3}+3 s^{2}+s, 0, s^{2}+s\right)$ в случае $s=4$ ?

1. Вспомогательные результаты. В этом разделе приведены некоторые вспомогательные результаты, используемые в дальнейшем.

ЛЕмма 1.1. Пусть Г - связный граф без 3-лап, $a \in \Gamma$. Тогда верны следующие утверждения:

(1) если $b, c$-несмежные вершины из $[a]$, то $[a] \subseteq b^{\perp} \cup c^{\perp}$;

(2) если $\{a, b ; c, d\}$ - четырехугольник из $\Gamma$, то $a^{\perp} \cup b^{\perp}=c^{\perp} \cup d^{\perp}$;

(3) если $\{a, b ; c, d\}$ - четырехугольник из Г и $\mu$-подграфы $[a] \cap[b],[c] \cap[d]$ являются регулярными графами одинаковой валентности, то $k_{a}+k_{b}=k_{c}+k_{d}, \mu_{a b}=$ $\mu_{c d}$.

ДокАЗАтЕЛьство. Если вершина $x \in[a]$ не лежит в $b^{\perp} \cup c^{\perp}$, то графф $\{a ; b, c, x\}$ является 3 -лапой и утверждение (1) доказано.

Пусть $\{a, b ; c, d\}$ - четьрехугольник из Г. По утверждению (1) $c^{\perp}$ и $d^{\perp}$ содержатся в $a^{\perp} \cup b^{\perp}$. Симметрично, $a^{\perp}$ и $b^{\perp}$ содержатся в $c^{\perp} \cup d^{\perp}$. Утверждение (2) доказано.

Пусть $\alpha$ - валентность указанных $\mu$-графов. По утверждению (1) $k_{a}=2+\lambda_{a c}+$ $\lambda_{a d}-\alpha, k_{b}=2+\lambda_{b c}+\lambda_{b d}-\alpha$. Вьписав аналогичные выражения для $k_{c}, k_{d}$, получим первое равенство в (3). Число вершин в графе $a^{\perp} \cup b^{\perp}$ равно $2+k_{a}+k_{b}-\mu_{a b}$. Это же число по утверждению (2) равно $2+k_{c}+k_{d}-\mu_{c d}$. Отсюда следует второе равенство в (3).

ЛЕмма 1.2. Если Г является регулярным графом степени $k$ без 3-коклик на $v$ вериинах, то Г кореберно регулярен с $\mu=2 k+2-v$.

ДокАЗАТЕЛЬСТво. Пусть $a \in \Gamma$. По условию $Г-a^{\perp}$ является $(v-k-1)$-кликой. Если $b \in \Gamma-a^{\perp}$, то $[b]$ содержит $v-k-2$ вершин из $\Gamma-a^{\perp}$ и $k-(v-k-2)$ вершин из $[a]$. Поэтому Г является кореберно регулярным графом с $\mu=2 k+2-v$.

ЛЕмма 1.3. Пусть Г- связный граф без 3-коклик на $v$ вериинах, в котором $\mu$-подграфы регулярны заданной положительной степени $\alpha$. Тогда выполняются следующие утверэдения:

(1) если $d(a, b)=2$, то подграф $[a] \cap[b]$ кореберно регулярен $c \mu^{\prime}=2 \alpha+2-\mu$, əде $\mu=\mu_{a b}$; 
(2) если b, с-несмежные вершины из $[a], \mu=\mu_{b c}$, то $[b] \cap[c] \cap \Gamma_{2}(a)$ является регулярным графом степени $k_{b}+k_{c}-k_{a} u \mu=\mu_{a x}$ для любой вершины $x$ из $\Gamma_{2}(a) \cap[b] \cap[c]$.

ДокАЗАТЕЛЬСТво. По условию $[a] \cap[b]$ является регулярным графом степени $\alpha$ на $\mu$ вершинах без 3-коклик. По лемме $1.2[a] \cap[b]$ является кореберно регулярным графом с $\mu^{\prime}=2 \alpha+2-\mu$. Утверждение (1) доказано.

Пусть $b, c$ - несмежные вершины из $[a], \mu=\mu_{b c}$. Для любой вершины $x$ из $\Gamma_{2}(a) \cap$ $[b] \cap[c]$ по лемме 1.1 верны равенства $k_{a}+k_{x}=k_{b}+k_{c}, \mu=\mu_{a x}$.

Лемма 1.4. Пусть Г - связный граф без 3-коклик, содержащий такой пятиугольник $\Delta$, что $[x] \cap[y]$ является $\mu$-кликой для любых двух несмежных вершин $x, y$ из $\Delta$. Тогда $k_{x}=k u \lambda_{x z}=(k-2+\mu) / 2$ для любых смехних вершин $x, z$ из $\Delta$.

ДокАЗАТЕЛЬСТво. По условию $v=2+k_{x}+k_{y}-\mu$ для любых двух несмежных вершин $x, y$ из $\Delta$. Пусть $a b c d e$ является 5-циклом из $\Delta$. Так как $v=2-\mu+k_{a}+$ $k_{c}=2-\mu+k_{a}+k_{d}$, то $k_{c}=k_{d}$. Двигаясь по циклу, получим, что $k_{x}=k$ для любой вершины $x$ из $\Delta$.

Теперь $k_{a}=2+\lambda_{a b}+\lambda_{a e}-\mu, k_{b}=2+\lambda_{a b}+\lambda_{b c}-\mu$, поэтому $\lambda_{a e}=\lambda_{b c}$. Аналогично $\lambda_{d e}=\lambda_{b c}$ и $k=k_{e}=2+\lambda_{a e}+\lambda_{d e}-\mu$, поэтому $\lambda_{a e}=(k-2+\mu) / 2$.

Лемма 1.5. Связный редуиированный граф Тервиллигера без 3-коклик является пятиугольником.

ДокАЗАТЕЛЬСТво. По условию Г не содержит вершин с кликовыми окрестностями и вершин $a$ таких, что $\Gamma=a^{\perp}$. Поэтому по теореме 1 [4] $Г$ является связным графом c $\mu=1$.

Пусть $a \in \Gamma, b, c$ - несмежные вершины из $[a]$. По редуцированности графра $[b]-a^{\perp}$ содержит вершину $d,[c]-a^{\perp}$ содержит вершину $e$. Теперь подграф $\Delta=\{a, b, d, e, c\}$ является пятиугольником. По лемме $1.4 k_{x}=k$ для любой вершины $x$ из $\Delta$.

Теперь по связности Г является регулярным графом. По лемме 1.1.3 [5] Г является сильно регулярным графом. По теореме Тэйлора-Ливингстона $[5,1.5 .3]$ сильно регулярный граф Тервиллигера без 3-лап является пятиугольником.

Лемма 1.6. Пусть Г - связный редуцированный граф без 3-коклик, в котором $\mu$-подграфы регулярны заданной положительной степени $\alpha$. Если Г содержит четырехугольник $\{a, b ; c, d\}, \quad \mu=\mu(a, b), \quad \Delta=[a] \cap[b] \cap[c] \cap[d]$, то выполняются следующие утверждения:

(1) максимальное значение $\mu$ равно $2(\alpha+1)$ и достигается тогда и только тогда, когда $\Delta$ nусто;

(2) если $\Delta$ не пусто $u[a] \cap[b]=\{c, d\} \cup \Delta$, то $[a] \cap[b]-$ полный многодольный граф $K_{r \times 2}$, где $\mu=2 r=\alpha+2$;

(3) если $([a] \cap[b])-(\{c, d\} \cup \Delta)$ содержит вершину е, без ограничения общности $e \in[c]-[d]$, mo $k_{c}=k_{e}, \lambda_{c e}=\lambda_{a c}=\lambda_{a e}=\lambda_{b c}=\lambda_{b e} ;$

(4) если $\alpha+3 \leqslant \mu \leqslant 2 \alpha+1$, то степени всех вершин из $\{a, b, c, d\}$ равны $k u$ $\lambda_{x y}=\lambda$ для любых смехнных вершин $x, y$ из $\{a, b, c, d\}$.

ДоКАЗАТЕЛЬСТВО. По лемме $1.3 \mu \leqslant 2 \alpha+2$, причем равенство достигается тогда и только тогда, когда $\Delta$ пусто. Утверждение (1) доказано. 
Пусть $\Delta$ не пусто и $[a] \cap[b]=\{c, d\} \cup \Delta$. Тогда $\mu=\alpha+2$, поэтому $[a] \cap[b]-$ полный многодольный граф $K_{r \times 2}$, где $\mu=2 r$. Утверждение (2) доказано.

Пусть $([a] \cap[b])-(\{c, d\} \cup \Delta)$ содержит вершину е и $e \in[c]-[d]$. Ввиду леммы 1.1 имеем $k_{c}=k_{e}$. Так как $k_{a}+\alpha-2=\lambda_{a d}+\lambda_{a c}=\lambda_{a d}+\lambda_{a e}$, то $\lambda_{a c}=\lambda_{a e}$. Аналогично, $k_{e}+\alpha-2=\lambda_{b e}+\lambda_{a e}=\lambda_{b e}+\lambda_{c e}$, поэтому $\lambda_{a e}=\lambda_{c e}$. Симметрично, $\lambda_{c e}=\lambda_{b c}=\lambda_{b e}$.

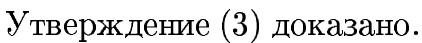

Пусть $\alpha+3 \leqslant \mu \leqslant 2 \alpha+1$. Тогда $([a] \cap[b])-(\{c, d\} \cup \Delta)$ содержит вершину $е$ из $[c]-[d]$ и вершину $f$ из $[d]-[c]$. Ввиду утверждения (3) $\lambda_{a c}=\lambda_{c b}=\lambda, \lambda_{a d}=\lambda_{d b}=\lambda^{\prime}$. Симметрично, $([c] \cap[d])-(\{a, b\} \cup \Delta)$ содержит вершину $g$ из $[a]-[b]$ и $\lambda_{a c}=\lambda_{a d}$. Значит, $\lambda=\lambda^{\prime}$.

Для $x \in\{a, b, c, d\}$ имеем $k_{x}=2 \lambda+2-\alpha$.

ЛЕмма 1.7. Пусть Г-сильно регулярный граф $с \mu=1$. Тогда окрестность каждой вериины из Г состоит из $r$ изолированных s-клик и выполняются следующие утверждения:

(1) $s+1<r, s+1$ делит $r(r-1)^{2}$; для $s=c^{2} f$, әде $f$ свободно от квадратов, получим $r-1=(t+c) t f$ u $2 t+c$ делит многочлен $R(s)$, где $R(s)=c s(s-$ $4)\left(s^{2}-2 s-4\right)$, если $s$ не делится на $4, u R(s)=c s(s-4)\left(s^{2}+2 s+4\right) / 16$, если $s$ делится на 4 ;

(2) параметр s не равен 2 и если $s=3$, то $r=7$;

(3) если $r \leqslant 6$, то Г является пятиугольником или графом Петерсена.

ДокАЗАТЕЛЬСтво. Пусть Г является сильно регулярньм графом с $\mu=1$. Тогда окрестность каждой вершины из $Г$ состоит из $r$ изолированных $s$-клик для некоторых натуральных чисел $r, s$.

По теореме 4 [6] $s+1 \leqslant r$ и вьполняются оставшиеся утверждения из (1). Покажем, что $s+1 \neq r$. В противном случае $t(t+c)=c^{2}$, но это уравнение не имеет целочисленных решений. Утверждение (1) доказано.

Пусть $s=2$. Тогда $c=1, f=2$ и $R(2)=16$. Противоречие с тем, что $2 t+1$ делит $R(s)$. Пусть $s=3$. Тогда $c=1, f=3$ и $R(3)=3$. Так как $2 t+1$ делит $R(s)$, то $t=1$ и $r-1=6$. Утверждение (2) доказано.

Если $r \leqslant 6$, то по утверждению $(1) s \leqslant 4$. Пусть $s=4$. Тогда $c=2, f=1$ и $R(4)=0$. Далее, $r-1=t(t+2)>4$, поэтому $t \geqslant 2$ и $r \geqslant 9$; противоречие. Ввиду утверждения (2) $s=1$ и Г является пятиугольником или графом Петерсена.

ЛЕмма 1.8. Пусть Г- сильно регулярный граф без 3-коклик с параметрами $(v, k, \lambda, \mu), \quad k>\lambda+1$. Тогда для каждой вершины $c \in \Gamma$ подграф $\Sigma=\Gamma(c)$ редуцирован.

ДокАЗАТЕЛЬСтво. По лемме 1.3 подграф $\Sigma$ является кореберно регулярным с $\mu(\Sigma)=2 \lambda+2-k$. Поэтому $\mu$-подграфы из $Г$ регулярны степени $\alpha=2 \lambda+2-k$.

Допустим, что граф $\Sigma$ не редуцирован. Так как $\Sigma$-регулярньй граф, то в $\Sigma$ найдутся такие две вершины $d, e$, что $d^{\perp} \cap \Sigma=e^{\perp} \cap \Sigma$. По сильной регулярности Г подграф $\Gamma(c) \cap \Gamma(d)=\Gamma(d) \cap \Gamma(e)$ содержит ровно $\lambda$ вершин. Отсюда следует, что подграфы $\Gamma(d)-\Gamma(c)$ и $\Gamma(e)-\Gamma(c)$ не пересекаются, и $\Gamma_{2}(c)$ содержит не меньше $2 b_{1}$ вершин, где $b_{1}=k-\lambda-1$.

Возьмем теперь две несмежные вершины $f, g \in \Sigma$. Поскольку $Г$ не содержит 3-коклик, имеет место равенство $\Gamma=f^{\perp} \cup g^{\perp}$. Но тогда подграфы $\Gamma(f)-\Gamma(c)$ и $\Gamma(g)-\Gamma(c)$ покрывают $\Gamma_{2}(c)$, и в $\Gamma_{2}(c)$ содержится не больше $2 b_{1}$ вершин. Из доказанного в предыдущем 
абзаце теперь следует, что $\left|\Gamma_{2}(c)\right|=2 b_{1}$, и подграфы $\Gamma(f)-\Gamma(c), \Gamma(g)-\Gamma(c)$ не пересекаются. Это означает, что $\mu$-подграф $\Gamma(f) \cap \Gamma(g)$ содержится в $c^{\perp}$ и $\alpha=\mu-1$. Последнее равенство означает, что $\Gamma$ - граф Тервиллигера. Но по лемме 1.5 сильно регулярньй граф Тервиллигера без 3-коклик - это пятиугольник. Противоречие с тем, что граф $\Sigma$ не редуцирован. Лемма доказана.

2. Доказательство предложения. Пусть Г является слабо редуцированным графом без 3-коклик, в котором $\mu$ подграфы регулярны степени $\alpha, \alpha>0$. До конца раздела предполагаем, что $b^{\perp} \subset a^{\perp}$ для некоторых вершин $a, b \in \Gamma$. Среди вершин $x$ со свойством $x^{\perp} \subset a^{\perp}$ выберем вершину $b$ наибольшей степени.

ЛЕмма 2.1. Выполняются следующие утверждения:

(1) если вершина $x$ из $[b]-\{a\}$ смехсна с вершиной вне $a^{\perp}$, то $[x]$ не пересекает $[a]-b^{\perp}$

(2) подграф $[b]$ не является кликой.

ДокАЗАтЕльство. Если вершина $x$ смежна с вершиной $c$ вне $a^{\perp}$ и вершиной $d$ из $[a]-b^{\perp}$, то $c, d$ смежны. Далее, в графе $[b] \cap[c]$ степень вершины $x$ равна $\alpha$. Противоречие с тем, что в графе $[a] \cap[c]$ вершина $x$ смежна еще $c d$. Утверждение $(1)$ доказано.

Пусть $[b]$ - клика. Тогда для любой вершины $d$ из $[a]-b^{\perp}$ подграф $[b] \cap[d]$ является $(\alpha+1)$-кликой. Пусть $x \in([b] \cap[d])-\{a\}$. В силу выбора вершины $b$ подграф $[x]$ не содержится в $a^{\perp}$; противоречие с утверждением (1).

ЛЕмма 2.2. Для любых двух несмежнных вершин $c, d$ из $[b]$ подграфы $[c] u[d]$ пересекают $[a]-b^{\perp}$.

ДоказАтельство. Допустим, что $c, d$ - несмежные вершины из $[b]$ и $[d]$ не пересекают $[a]-b^{\perp}$. Тогда $[a]-b^{\perp} \subset[c]$. Для $e \in[a]-b^{\perp}$ подграф $[d] \cap[e]$ содержит $\alpha+1$ вершин из $a^{\perp}$, попадающих в [b]. Противоречие с тем, что в графе $[b] \cap[e]$ вершина $a$ смежна еще с вершиной $c$.

ЛЕмма 2.3. Выполняются следующие утверждения:

(1) $\Gamma^{\perp}=\{a\}$ и Г является графом Тервиллигера;

(2) если $c, d$-несмежнные вершины $[b]$, то $[b]=\{a, c, d\}$;

(3) Г является пятиугольной пирамидой.

ДокАЗАТЕЛЬСтво. Заметим, что по лемме 2.2 окрестности любых двух несмежных вершин из $[b]$ попадают в $a^{\perp}$. Поэтому $\Gamma=a^{\perp}$. Из слабой редуцированности следует, что $\Gamma^{\perp}=\{a\}$. Теперь степень вершины $a$ в $\mu$-подграфе равна $\alpha$, поэтому указанньй $\mu$-подграф является $(\alpha+1)$-кликой. Утверждение (1) доказано.

Пусть $c, d$-несмежные вершины $[b], e \in[c] \cap\left([a]-b^{\perp}\right), f \in[d] \cap\left([a]-b^{\perp}\right)$. Так как $\Gamma$ - граф Тервиллигера, то $[c] \cap[d]$ не пересекает $[a]-b^{\perp}$. Далее, для любой вершины $x$ из $[e] \cap([b]-\{a\})$ верно включение $[e] \subset c^{\perp}$. Действительно, $[c] \cap\left([a]-b^{\perp}\right)=[x] \cap\left([a]-b^{\perp}\right)$, и если $y \in[b]-c^{\perp}$, то $[f] \cap[x]$ не является кликой. По слабой редуцированности графа имеем $[e] \cap([b]-\{a\})=\{c\}$. Симметрично, $[f] \cap([b]-\{a\})=\{d\}$ и степень вершины $b$ равна 3. Утверждение (2) доказано.

Теперь $f^{\perp}=g^{\perp}$ для любой вершины $g$ из $[d] \cap\left([a]-b^{\perp}\right)$, поэтому $\left|[d] \cap\left([a]-b^{\perp}\right)\right|=1$. Симметрично, $\left|[c] \cap\left([a]-b^{\perp}\right)\right|=1$ и $[a]$ является пятиугольником. Лемма, а вместе с ней и предложение доказаны. 
3. Графы Тервиллигера без корон. В этом разделе будут доказаны теорема 1 и следствие 1.

Лемма 3.1. Пусть Г - связный граф Тервиллигера без 3-коклик. Тогда Гявляется кликовым расширением одного из следуюших графов:

(1) 2-пути или 3-пути, причем порядок клики, заменяющей не концевую вершину пути, равен $\mu$;

(2) пятиугольника или пятиугольной пирамидь, причем порядок клики, заменяющей вершину пятиугольника, равен $\mu$.

ДокАЗАТЕЛЬСтво. По теореме 1 [4] либо $\Gamma-\Gamma^{\perp}$ содержит вершину $а$ с кликовой окрестностью, либо $\Gamma-\Gamma^{\perp}$ является кликовьм расширением редуцированного граба.

В первом случае либо диаметр Г равен 3 и Г является кликовьм расширением 3-пути, либо диаметр Г равен 2 и Г является кликовьг расширением 2-пути. Ясно, что порядок клики, заменяющей не конщевую вершину пути, равен $\mu$, и утверждение (1) вьполняется.

Во втором случае по лемме $1.5 \Gamma-\Gamma^{\perp}$ является кликовым расширением пятиугольника, и утверждение (2) вьполняется.

Лемма 3.2. Пусть Г - связный граф Тервиллигера без 3-лап, содержсаший 3-коклику, $\Gamma^{\prime}$ - подграфна множестве вершин из Г с некликовыми окрестностями. Тогда диаметр Г больше 2 и выполняется одно из следующих утверждений:

(1) Г является кликовым расширением графа икосаэдра;

(2) $\Gamma^{\prime}-$ клика $и$ Г является кликовым расширением графа $\Delta$, где $\Delta$ содержит $\delta$-клику и $\delta$ или $\delta-1$ вершин степени 1 , смежнны с различными вершинами кликu;

(3) $\Gamma^{\prime}$ является кликовым расширением графа с $\mu=1$.

ДокАЗАТЕЛьСтво. По теореме 2 [4] диаметр Г больше 2. По условию $\Gamma^{\perp}-$ пустой граф. Пусть $\Gamma^{\prime}$ - подграф на множестве вершин из Г с некликовыми окрестностями.

По теореме 1 [4] либо Г является кликовым расширением графа икосаэдра, либо $\Gamma^{\prime}$ является кликовым расширением клики или графа с $\mu=1$. По лемме 3.1 для $b \in \Gamma^{\prime}$ подграф $[b]$ является кликовым расширением 2-пути, пятиугольника или пятиугольной пирамиды.

Пусть $\Gamma^{\prime}$ является кликой. Тогда $Г$ содержит две несмежных вершины $a, c$ с кликовыми окрестностями. Если $[a] \cap[c]$ содержит вершину $b$, то $\Gamma^{\prime} \subset a^{\perp} \cup c^{\perp}$ и $\Gamma-b^{\perp}$ содержит вершину $d$ с кликовой окрестностью. Заметим, что $[a] \cap[d]$ и $[c] \cap[d]$ содержатся в $b$, и если указанные $\mu$-подграфы не пусты, то они совпадают с $[b] \cap[d]$. Противоречие с тем, что окрестность вершины из $[b] \cap[d]$ содержит 3 -коклику $a, c, d$. Поэтому один из этих подграфов, скажем, $[c] \cap[d]$, пуст и $\Gamma^{\prime} \subset[a]$.

Пусть подграфы $[a],[c]$ не пересекаются для любых несмежных вершин $a, c \in \Gamma-\Gamma^{\prime}$. Тогда $[b] \cap[c]$ является $\mu$-кликой из $\Gamma^{\prime}$ для $b \in \Gamma^{\prime}-[c]$.

Итак, если $\Gamma^{\prime}$ является кликой, то $\Gamma$ является кликовым расширением графа $\Delta$, где $\Delta$ содержит $\delta$-клику и $\delta$ или $\delta-1$ вершин степени 1 , смежных с различными вершинами клики. 
Лемма 3.3. Пусть Г-связный граф Тервиллигера без корон, содержащий 3-лапу. Тогда $\mu=1$ и окрестность кажсдой вериины из $Г$ состоит из изолированных клик.

ДокАЗАТЕЛЬСТво. Если $\mu>1$, то окрестность каждой вершины из Г является графом Тервиллигера диаметра 2 без 3-лап, причем окрестность некоторой вершины содержит 3-коклику. Противоречие с леммой 3.2 .

Значит, $\mu=1$. Теперь окрестность каждой вершины состоит из изолированных клик. Лемма доказана.

Теорема 1 следует из лемм 3.1-3.3.

Лемма 3.4. Пусть Г-связный граф, в котором окрестности вериин являются регулярными графами Тервиллигера диаметра 2. Если окрестность некоторой вериины не содержит 7-лап, то либо Г является кликовым расширением графа икосаэдра, либо окрестность любой вершины из Г является $\beta$-расширением графа Петерсена.

ДокАЗАТЕЛЬСТво. Пусть $a \in \Gamma, \Sigma_{0}=[a]$. По предложению $1.16 .2[5] \Sigma_{0}$ является $\beta_{0}$-расширением сильно регулярного графа Тервиллигера $\Sigma_{1}$.

Допустим, что $\Sigma_{0}$ не содержит 7-лап. Снова по предложению 1.16 .2 [5] окрестность любой вершины в графе $\Sigma_{1}$ является $\beta_{1}$-расширением сильно регулярного графа Тервиллигера $\Sigma_{2}$, и так далее. Таким образом, для некоторого $l \geqslant 0$ граф $\Sigma_{l}$ является $\beta_{l}$-расширением сильно регулярного графа с $\mu=1$. Так как $\Sigma_{l}$ не содержит 7 -лап, по лемме $1.7 \Sigma_{l}$ является кликовьм расширением пятиугольника или графа Петерсена.

Если $l>0$, то по теореме 1.16.3 [5] $s=\beta_{l} \leqslant 1$, поэтому $\Sigma_{l}$ является пятиугольником или графом Петерсена. Связньй локально пятиугольньй граф является графом икосаэдра и имеет диаметр 3. По теореме 1.16.5 [5] связньй локально Петерсеновский граф является дополнительным графом для треугольного графа $T(7)$, графом Конвея-Смита на 63 вершинах диаметра 4 (это антиподальное 3 -накрытие $\overline{T(7)}$ ) или графом Доро на 65 вершинах диаметра 3. Противоречие с тем, что $\Sigma_{l-1}$ является графом Тервиллигера диаметра 2. Значит, $l=0$.

Пусть $b \in \Sigma_{0}, \Sigma^{\prime}=\Gamma(b)$. Если $\Sigma_{0}$ содержит 3-лапу, то $\Sigma^{\prime}(a)$ является кликовым расширением 3-лапы и $\Sigma^{\prime}$ является кликовым расширением графа Петерсена. По связности графа окрестность любой вершины является $\beta_{0}$-расширением графа Петерсена.

Если же $\Sigma_{0}$ не содержит 3 -лап, то $\Sigma^{\prime}(a)$ является кликовым расширением 2-пути и $\Sigma^{\prime}$ является кликовым расширением пятиугольника. Снова по связности графа окрестность каждой вершины из Г является кликовым расширением пятиугольника и по теореме 1 Г является $\beta$-расширением графа икосаэдра. Лемма доказана.

Допустим, что окрестность некоторой вершины из Г не содержит корон. По теореме 1.16 .5 [5] связньй локально Петерсеновский граф является дополнительным графом для треугольного графа $T(7)$, графом Конвея-Смита на 63 вершинах диаметра 4 или графом Доро на 65 вершинах диаметра 3. Следствие 1 доказано.

4. Редуцированные графы без 3-коклик. В этом разделе мы докажем теорему 2 и следствие 2 . Пусть $Г$ - связный редуцированньй граф без 3-коклик, в котором каждьй $\mu$-подграф регулярный заданной положительной степени $\alpha$. Допустим, что $\Gamma$ не является сильно регулярньм графом. Если $Г$ - граф Тервиллигера, то по теореме 1 Г является пятиугольником. Значит, Г содержит четырехугольник. 


\section{ЛЕмма 4.1. Г не содержит несвязных $\mu$-подграфов.}

ДокАЗАТЕЛЬСтво. Пусть граф $[a] \cap[b]$ несвязен. Тогда он состоит из двух изолированных клик $C, D$ порядка $\alpha+1$. Пусть $c, e-$ две вершины из $C$. Тогда графы $c^{\perp}$, $e^{\perp}$ совпадают вне $d^{\perp}$ для любой вершины $d \in D$. Выберем вершину $x \in[c]-e^{\perp}$. Без ограничения общности, $x \in[a] \cap D^{\perp}$. Заметим, что $[x] \cap[b]$ содержит изолированную клику $D$, поэтому $[x] \cap[b] \cap c^{\perp}$ лежит в $e^{\perp}$. Но тог да в графе $[e] \cap[x]$ вершина $c$ смежна еше с $a$ и валентность $c$ в этом графе не меньше $\alpha+1$. Значит, $c^{\perp}=e^{\perp}$, что противоречит редуцированности графа.

ЛЕмма 4.2. Для любых двух несмежсных вериин $a, b$ получим $\mu_{a b}=\alpha+1$ или $\alpha+2$.

ДоказАтельство. Допустим, что $\mu_{a b}>\alpha+2$. Выберем несмежные вершины $c, d$ из $[a] \cap[b]$. Из лемм 1.6, 4.1 следует, что степени всех вершин из $\{a, b, c, d\}$ равны $k$ и $\lambda=\lambda_{x y}$ для любых смежных вершин $x, y$ из $\{a, b, c, d\}$.

Покажем, что степень любой вершины $x$ из Г равна $k$. Если $x \in[c] \cap[d]$, то $k_{x}=k$ по лемме 1.1. Пусть $e \in([c]-[d])-a^{\perp}$ и $k \neq k_{e}$.

Ввиду леммы $1.6 \mu_{a e}=\alpha+1$ или $\alpha+2$. Пусть $\Gamma^{\prime}$-подграф, индуцированньй на множестве всех вершин из Г степени $k$. Тогда $\mu\left(\Gamma^{\prime}\right)=\mu_{a b}$ и $\Gamma^{\prime}$ является сильно регулярным графом, содержащим геодезический 5-цикл $a_{1} \ldots a_{5}$. Пусть $e \in \Gamma-\Gamma^{\prime}$. Тогда $[e] \cap \Gamma^{\prime}$ является кликой. Без ограничения общности, $е$ не смежна с $a_{1}$. Тогда $e \in\left[a_{3}\right] \cap\left[a_{4}\right]$. Поэтому вершины $a_{2}, a_{5}$ не смежны с $e$; противоречие.

ЛЕмма 4.3. Подграф $\Gamma^{\prime}$, индуцированный на мнохсестве всех вершин а из Г таких, что $\mu(a, b)=\alpha+2$ для некоторой вершины $b \in \Gamma_{2}(a)$, является полным многодольным графом $K_{s \times 2}$, где $2 s=\alpha+4$.

ДоказАтЕльство. Так как Г не является графом Тервиллигера, найдутся несмежные вершины $a, b$ с $\mu_{a b}=\alpha+2$. Тогда $k_{a}+k_{b}-\alpha=v$ и $\mu_{c d}=\alpha+2$ для любых двух несмежных вершин $c, d \in[a] \cap[b]$.

Пусть $\Gamma^{\prime}$ - подграф, индуцированньй на множестве всех вершин $a$ из $\Gamma$ таких, что $\mu(a, b)=\alpha+2$ для некоторой вершины $b \in \Gamma_{2}(a)$. Если $\Gamma^{\prime}$ содержит пятиугольник, то $\Gamma^{\prime}$ является сильно регулярным графом. Повторив рассуждения из последнего абзаца леммы 4.2 , получим противоречие.

Значит, $\Gamma^{\prime}$ не содержит пятиугольников. Без ограничения общности, вершина $a$ из $\Gamma^{\prime}$ имеет наименьшую степень в $\Gamma$. Допустим, что $\Gamma^{\prime}(a)-[b]$ содержит вершину $c$. Тогда $[a] \cap[b] \cap[c]$ является $(\alpha / 2+1)$-кликой и $([b] \cap[c])-[a]$ содержит вершину $d$. Так как $\Gamma^{\prime}$ не содержит пятиугольников, то $([a] \cap[b])-[c] \subset[d]$. Поэтому $[b] \cap[c]$ не пересекает $[a]$ и является $(\alpha+1)$-кликой. Отсюда $k_{a}=k_{c}+1$; противоречие.

Таким образом, $\Gamma^{\prime}$ является полным многодольным графом $K_{s \times 2}$, где $2 s=\alpha+4$. Лемма доказана.

Завершим доказательство теоремы 2. Для вершины $a$ из $\Gamma^{\prime}$ через $a^{*}$ обозначим единственную вершину из $\Gamma^{\prime}-a^{\perp}$. Заметим, что для $e \in \Gamma-\Gamma^{\prime}$ подграф $[e] \cap \Gamma^{\prime}$ является $s$-кликой. Если $a \in \Gamma^{\prime}-[e]$, то $k_{a}+k_{e}+1-\alpha=v$. Поэтому $k_{b}=k_{e}+1$ для $b \in[e] \cap \Gamma^{\prime}$ и $[a] \cap[e]$ содержит $\alpha / 2$ вершин из $\Gamma-\Gamma^{\prime}$. Пусть $f \in[e] \cap\left(\Gamma-\Gamma^{\prime}\right)$. Тогда $[f] \cap \Gamma^{\prime}$ содержит $a$ и $s-1$ вершин из [e]. Поэтому $k_{e}=k_{f}=k_{a}-1$, подграф $\Gamma^{\prime}$ является регулярным степени $k$ и подграф $\Gamma-\Gamma^{\prime}$ является регулярньг степени $k-1$. В частности, подграф $\Gamma-\Gamma^{\prime}$ является кликой. 
Положим $\beta=\left|\Gamma-\Gamma^{\prime}\right|$. Тогда $k_{a}=\alpha+2+\beta / 2, k_{e}=\alpha / 2+2+(\beta-1)$, поэтому $\beta=\alpha+2$. Как показано выше, если $a \in \Gamma^{\prime}, e \in\left(\Gamma-\Gamma^{\prime}\right)-[a]$, то $\left([e] \cap \Gamma^{\prime}\right)-\left\{a^{*}\right\} \subset[f]$ для любой вершины $f \in\left(\Gamma-\Gamma^{\prime}\right) \cap[a]$. Симметрично, $\left([f] \cap \Gamma^{\prime}\right)-\{a\} \subset[g]$ для любой вершины $g \in\left(\Gamma-\Gamma^{\prime}\right) \cap\left[a^{*}\right]$. Противоречие с тем, что $\alpha \geqslant 1$. Теорема 2 доказана.

ДоКАЗАТЕЛЬСТВО СЛЕДСТВИЯ 2. Пусть Г - связньй редуцированный граф без корон, в котором каждый $\mu$-подграф $\Delta$ реберно регулярен с заданньми параметрами $\left(v^{\prime}, k^{\prime}, \lambda^{\prime}\right), k^{\prime}>0$, и имеет диаметр не больше 2 . Если $Г$ является регулярным графом Тервиллигера, то следствие 2 выполняется ввиду теоремы 1 . Если $Г$ не является графом Тервиллигера и содержит 3-коклику, то следствие выполняется по теоремам 1, 3 [2]. Допустим, что Г не содержит 3-коклик. По теореме 2 Г является сильно регулярньм графом без 3-коклик. Ввиду леммы 1.3 каждый $\mu$-подграф $\Delta$ сильно регулярен с $\mu(\Delta)=2 k^{\prime}+2-v^{\prime}$. По лемме 1.8 окрестности вершин в $Г$ являются редуцированньми графами. Пусть $\lambda^{\prime}>0, a \in \Gamma, \Sigma=\Gamma(a)$. Тогда $\Sigma$ является редуцированным графом без 3-коклик, в котором $\mu$-подграфы регулярны положительной степени $\lambda^{\prime}$. По теореме $2 \Sigma$ является сильно регулярным графом. По теореме 8.8 [6] $\Gamma$ - граф из заключения следствия. Пусть $\lambda^{\prime}=0$. Тогда окрестности вершин в $\Delta$ являются 2-кокликами, поэтому $\Delta$ является $m$-угольником для $m=4$ или 5 . Пусть $\Sigma$ является дополнительным графом для $Г$.

Если $\Delta$ является четырехугольником, то либо $Г$ является октаэдром, либо $\Sigma$ имеет параметры $(v, k, 0, \mu)$, где $v-2 k=4$. В последнем случае по прямоугольному соотношению $(k+3) \mu=k(k-1)$ и $k+3$ делит 12 . Поэтому $k=3$ или 9 ; соответственно, $\mu=1$ или 6. Если $\mu=1$, то $\Sigma$-граф Петерсена. Если же $\mu=6$, то $\mu^{2}+4(k-\mu)$ не является квадратом натурального числа; противоречие. Итак, если $\Delta$ является четырехугольником, то Г является октаэдром или треугольным графом $T(5)$.

Пусть $\Delta$ является пятиугольником. Тогда $\Sigma$ имеет параметры $(v, k, 0, \mu)$, где $v-$ $2 k=5$. В этом случае по прямоугольному соотношению $(k+4) \mu=k(k-1)$ и $k+4$ делит 20 . Поэтому $k=6$ или 16 ; соответственно $\mu=3$ или 12 . Противоречие с тем, что $\mu^{2}+4(k-\mu)$ должно быть квадратом натурального числа.

\section{СПИСОК ЦИТИРОВАННОЙ ЛИТЕРАТУРЫ}

[1] Numata M. A characterization of Grassman and Johnson graphs // J. Comb. Theory. Ser. B. 1990. V. 48. P. 178-190.

[2] Кабанов В. В. О графах без корон с регулярными $\mu$-подграфами // Матем. заметки. 2000. T. 67. №6. C. $874-881$.

[3] Махнев А. А. Об одном классе граффов без 3-лап // Матем. заметки. 1998. Т. 63. №3. C. $407-413$.

[4] Кабанов В. В., Махнев А. А. Графы без 3-лап с равномощными $\mu$-подграфами // Изв. Урал. гос. ун-та. Матем., мех. 1998. № 10. С. 44-68.

[5] Brouwer A. E., Cohen A. M., Neumaier A. Distance-Regular Graphs. Springer-Verlag, 1989.

[6] Cameron P., Van Lint J. Designs, Graphs, Codes and their Links // London Math. Soc. Student Texts 22. Cambridge: Cambr. Univ. Press, 1981.

Институт математики и механики УрО РАН,

г. Екатеринбург 